\title{
Variant of plasmablastic microlymphoma in Castleman disease: a case report and review of the literature
}

\author{
Nathan Artom, ${ }^{1}$ Marcello Brignone, ${ }^{1}$ Luca Paris, ${ }^{1}$ Anna Lisa Garlaschelli, ${ }^{1}$ Marina Cavaliere, ${ }^{1}$ Gian Luca Michelis, ${ }^{1}$ \\ Claudia Venturino, ${ }^{1}$ Silvia Ardoino, ${ }^{2}$ Ezio Venturino, ${ }^{2}$ Paola Gnerre,${ }^{1}$ Rodolfo Tassara ${ }^{1}$ \\ ${ }^{1}$ Department of Internal Medicine; and ${ }^{2}$ Department of Clinical Pathology, San Paolo Hospital, Savona, Italy
}

\begin{abstract}
Castleman disease (CD) is a rare lymphoproliferative disorder also known as angiofollicular lymph node hyperplasia or giant lymph node hyperplasia. $\mathrm{CD}$ can be unicentric $\mathrm{CD}$ (UCD) or multicentric $\mathrm{CD}$ (MCD). MCD affects more than one group of lymph nodes and/or lymphoid tissues and it is frequently associated with HIV and human herpes virus 8 (HHV-8) infections and, in contrast with UCD, it often results in systemic symptoms, such as fever, fatigue, anemia, inflammatory syndrome. HHV8-associated MCD recognizes HHV-8 as an etiopathogenetic agent and occurs generally in HIV-positive subjects. Our report describes an HHV-8 positive Castleman disease with plasmablastic microlymphoma occurring in a 51-year-old HIV seronegative woman, with a previous history of HBV infection and Kaposi's sarcoma, who presented elevated procalcitonin levels during the acute phase of CD.
\end{abstract}

\section{Introduction}

Castleman disease (CD) is a rare lymphoproliferative disorder also known as angiofollicular lymph node hyperplasia or giant lymph node hyperplasia. ${ }^{1} \mathrm{CD}$ is characterized by hyperplastic lymph nodes with hyalinized foci and was described for the first time in 1956 by Sir Benjamin Castleman, who reported 13 cases of mediastinal mass in asymptomatic patients. ${ }^{2}$ Indeed, $\mathrm{CD}$ includes heterogeneous types of disorders. ${ }^{3,4}$ According to the distribution of the disease, in 1978 Gaba distinguished between unicentric CD (UCD) and multicentric $\mathrm{CD}$ (MCD) ${ }^{3,4} \mathrm{MCD}$ affects more than one group of lymph nodes and/or lymphoid tissues and it is

Correspondence: Paola Gnerre, Department of Internal Medicine, San Paolo Hospital, via Genova I, 17100 Savona, Italy. Tel.: +39.0198404358 - Fax: +39.0198404583.

E-mail:pgnerre@yahoo.it

Key words: Castleman disease; lymphoproliferative disorder; multicentric Castleman disease.

Conflict of interest: the authors declare no conflict of interest.

Received for publication: 1 July 2018.

Revision received: 27 August 2018.

Accepted for publication: 19 September 2018.

This work is licensed under a Creative Commons Attribution NonCommercial 4.0 License (CC BY-NC 4.0).

CCopyright N. Artom et al., 2018

Licensee PAGEPress, Italy

Italian Journal of Medicine 2018; 12:276-279

doi:10.4081/itjm.2018.1063 frequently associated with HIV and human herpesvirus 8 (HHV-8) infections and, in contrast with UCD, it often results in systemic symptoms, such as fever, fatigue, anemia, inflammatory syndrome. ${ }^{4}$

Histologically, there are four variants of $\mathrm{CD}$ : hyaline vascular type (the most common variant), plasma cell CD, HHV-8-associated CD (plasmablastic variant) and MCD not otherwise specified. ${ }^{5}$ The plasmablastic variant recognizes $\mathrm{HHV}-8$ as an etiopathogenetic agent, it occurs generally in HIVpositive subjects, ${ }^{4}$ and presents $\mathrm{HHV}-8$ positive immunoblastic-like cells (plasmablasts) in the mantle zone of B-cells follicles. These cells express CD27, $\mu$ heavy chain (M) and $\lambda$ light chain of the immunoglobulin (Ig), typically in a monotypic fashion in spite of a polyclonal pattern of Ig gene rearrangement. In a particular subtype of HHV-8associated MCD, more numerous large cells with plasmacytoid features (plasmablasts) form microscopic aggregates either adjacent to or partially replacing the lymphoid follicles, this subtype is named plasmablastic microlymphoma. ${ }^{5,6}$

Our report describes an HHV-8 positive Castleman disease with plasmablastic microlymphoma occurring in a 51-year-old HIV seronegative woman, with a previous history of hepatitis B virus (HBV) infection and Kaposi's sarcoma (KS), who presented elevated procalcitonin $(\mathrm{PCT}$ ) levels during the acute phase of $\mathrm{CD}$.

\section{Case Report}

A 51-year-old Caucasian female came to our observation because of persistent intermittent fever, asthenia and joint pain despite 7 days of amoxicillin 
clavulanate followed by 7 days of levofloxacin. Her medical history included a previous HBV infection and, 1 year before the admission to our ward, a KS localized to the right ankle treated with surgical excision and then subjected to oncological follow-up. The physical examination showed right cervical, axillary lymph nodes enlargement and splenomegaly. Blood tests demonstrated hemoglobin $8.3 \mathrm{~g} / \mathrm{dL}$, platelets $9000 / \mu \mathrm{L}$, lactate dehydrogenase (LDH) 390 $\mathrm{U} / \mathrm{L}, \mathrm{C}$-reactive protein $25.7 \mathrm{mg} / \mathrm{dL}$ (normal values $<0.5 \mathrm{mg} / \mathrm{dL}$ ), PCT $57.77 \mathrm{ng} / \mathrm{mL}$ (normal values $<0.05$ $\mathrm{ng} / \mathrm{mL}$ ). The patient was HIV-seronegative, whereas the qualitative real-time polymerase chain reaction test for HHV-8 in serum gave a positive result. The screening for $\mathrm{HbsAg}$ was positive and the other markers of HBV infection (HBsAb, HbeAg, $\mathrm{HbcAb}$, $\mathrm{HbeAb}$ ) were negative. The other laboratory tests were normal. We immediately treated the patient with red cells and platelets transfusion and performed an excisional biopsy of the right axillary lymph node and a 18F-fluorodeoxyglucose (FDG) positron-emission tomography-computed tomographic (PET-CT) scan. This last showed elevated standardized uptake value in supraclavicular, laterocervical, axillary, mediastinal, para-aortic, aorto-iliac lymph nodes and in spleen. Because of the intermittent fever and the high values of PCT we performed blood and urine cultures and started antibiotic therapy with piperacillin tazobactam as first line follow by vancomycin and meropenem. Methylprednisolone was used to improve symptoms. The lymph node biopsy showed cortical areas with atrophic but vascular germinal centers, with a good representation of the mantle zone. Interfollicular areas and the mantle zone of follicles presented aggregates of monotypic plasmablasts. These inter- e intrafollicular aggregates of plasmablasts were HHV8 positive and $\lambda$ light chain restricted; molecular analysis showed monoclonal IGH (Figures 1-3). These findings were consistent with a diagnosis of HHV-8 positive Castleman disease, hyaline-vascular variant, with numerous monotypic/monoclonal plasmoblasts, consistent with microlymphoma, in a HIV seronegative patient. Antibiotics were stopped when blood and urine cultures resulted negative. Because of persistent elevated fever and severe cytopenia not responsive to corticosteroid, the patient started a treatment with intravenous infusions of etoposide and rituximab. The dose of Etoposide was $100 \mathrm{mg} / \mathrm{m}^{2}$ given every two weeks for 4 administrations. Following completion of Etoposide, the dose of Rituximab was $375 \mathrm{mg} / \mathrm{m}^{2}$ once weekly for 4 weeks. This treatment determined an initially transient and then stable improvement. During chemotherapy prophylactic lamivudine was used. On day 45 after admission the patient was discharged. At that time, she was asymptomatic and laboratory findings were

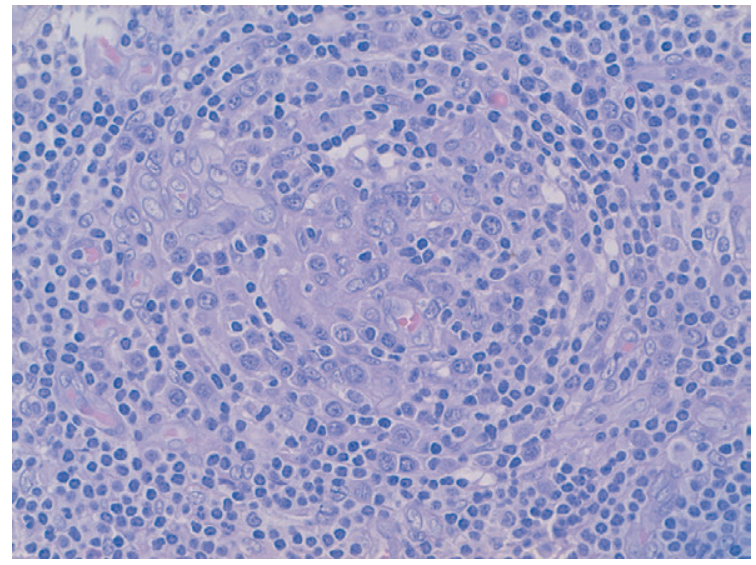

Figure 1. EE 40X. Medium-sized plasmablastic cells with amphophilic cytoplasm and vesicular nuclei with one or two prominent nucleoli, among mantle zone cells.

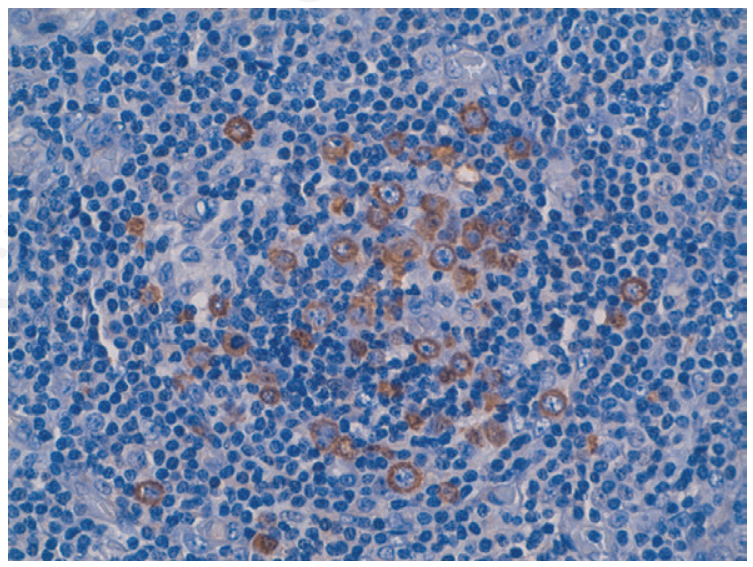

Figure 2. Lambda 40X. Plasmablastic cells: monotypic expression of $\lambda$ light chain.

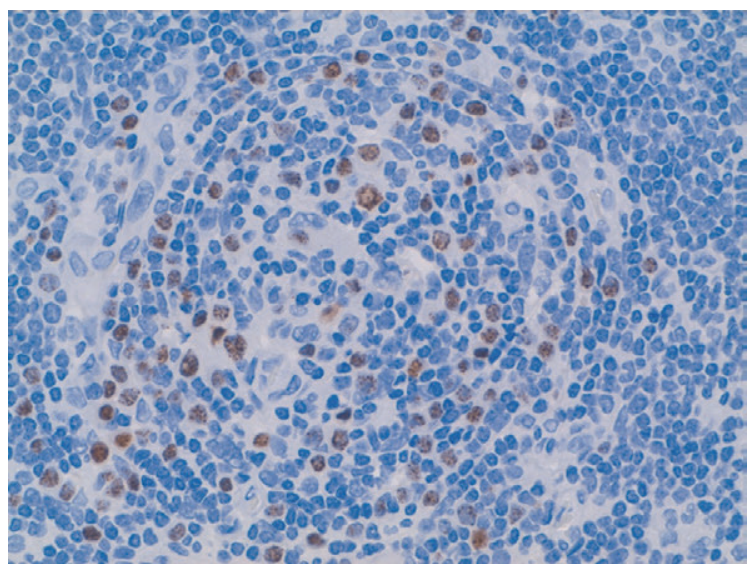

Figure 3. HHV8 40X. Plasmablastic cells HHV8+. 
hemoglobin $11.3 \mathrm{~g} / \mathrm{dL}$, platelets $110.000 / \mu \mathrm{L}, \mathrm{LDH} 211$ U/L, C-reactive protein $10.5 \mathrm{mg} / \mathrm{dL}$, PCT $3.18 \mathrm{ng} / \mathrm{mL}$. The other laboratory tests were normal. After 3 months the patient was still asymptomatic and biochemical data showed hemoglobin $15.3 \mathrm{~g} / \mathrm{dL}$, platelets $278.000 / \mu \mathrm{L}, \mathrm{LDH} 246 \mathrm{U} / \mathrm{L}, \mathrm{C}$-reactive protein $0.15 \mathrm{mg} / \mathrm{dL}$, PCT $<0.05 \mathrm{ng} / \mathrm{mL}$. Another 18 FDG PET-CT demonstrated the disappearance of lymphadenopathy and splenomegaly with normalized accumulation of FDG. These clinical, biochemical and radiological data were consistent with a complete remission of $\mathrm{CD}$.

\section{Discussion}

$\mathrm{CD}$ is a nonclonal lymphoproliferative disorder affecting single or many lymph node stations. ${ }^{1,4}$ In fact, according to the disease dissemination, $C D$ is divided into unicentric form and multicentric form. ${ }^{4}$ However, CD has the potential to affect any organ and thus presents with systemic manifestations.

HHV8-positive plasmablastic lymphoma is a rare subtype of the MCD, where plasmablasts become more numerous and constitute inter- and intrafollicular aggregates. ${ }^{6}$

Systemic manifestations such as constitutional symptoms (fever, night sweats, and malaise), hepatosplenomegaly, marked lymphadenopathy is common such as hematological (anemia and thrombocytopenia) and/or immunological abnormalities. ${ }^{1}$

The systemic symptoms have been associated with increased IL-6 levels, which are found elevated in the majority of cases. IL- 6 overproduction is often related to HHV8 infection. ${ }^{7} \mathrm{HHV}-8$ encodes for the viral orthologue of IL-6, which determines the release of human IL-6 and the subsequent cytokine storm responsible for the systemic symptoms. ${ }^{7}$ Because of its rarity, $\mathrm{CD}$ is inadequately understood and is unprovided of a unique international classification of disease code. The Castleman Disease Collaborative Network (CDCN) was built to study and better understand this rare disease. ${ }^{1}$ In fact, the incidence of all forms of CD has been estimated to be 6500-7700 subjects every year in the United States. ${ }^{8}$

Common therapeutic approaches for MCD include low-dose single-agent chemotherapy, such as etoposide or cladribine; combination chemotherapy, such as CHOP (cyclophosphamide, doxorubicin, vincristine, and prednisolone); monoclonal antibodies, like anti-CD20 antibody rituximab. ${ }^{9,10}$ Recently, siltuximab, a monoclonal antibody against IL-6 has been used successfully in MCD. ${ }^{11}$

We therefore reported a case of a 51-year-old, HIV-seronegative, immunocompetent female, with a past medical history of KS and $\mathrm{HBV}$ infection, who presented an MCD variant HHV8 plasmablastic microlymphoma with elevated PCT levels during the phase of the activation of the disease that mimic a sepsis. In fact, patient presented fever with chills, joint pain, elevated levels of C-reactive protein and PCT that, before the result of blood and urine cultures, required broad-spectrum antibiotics, because we were not able to exclude a concomitant sepsis in a patient immunosuppressed by her suspected hematological disease, especially considering PCT levels. In fact, PCT is well-known as a laboratory marker helpful for discriminating an infectious fever (in particular, bacterial) from a non-infectious one. ${ }^{12}$ Antibiotic therapy has been discontinued when blood cultures resulted negative. Recently, only Nara et al. ${ }^{13}$ reported two cases of MCD, without specifying which histological variant, with elevated PCT levels that mimic a septic condition.

Our patient presented a history of KS and HBV. ${ }^{14,15}$ Typically, CD in patients with KS are HIV and HHV8 positive. However, KS/HHV8 MCD can also occur in HIV-negative subjects, but the common variants are hyaline vascular and plasma cell type. From 1995 to 2012, Dossier et al. reported 18 cases of MCD in HHV-8 positive subjects not infected with HIV. Nine cases presented KS. However, in contrast with our patient, all 18 cases showed the classical histopathological features of CD. ${ }^{16}$ In 2012, Alkaied, and coworkers described the first case of MCD variant microlymphoma in a HHV-8 positive and HIV seronegative patient with a history of KS. ${ }^{17}$ About HBV and CD, Yuan and Collaborators showed an elevated prevalence of HBV infection in MCD in a group of HIV seronegative subjects, suggesting a pathogenetic role for $\mathrm{HBV}$ for the genesis of MCD in this subgroup of patients. ${ }^{15}$

In the available literature the incidence of HHV-8 plasmablastic microlymphoma is very rare, and in HIV seronegative patients is anecdotal: besides Alkaied report, Koenig and coworkers presented a similar case in a 67-year-old Caucasian female, ${ }^{18}$ while Lee and collaborators discussed a case of HHV8/EBV copositive plasmoblastic microlymphoma in a 53-yearold immunocompetent man. ${ }^{19}$

Because of MCD rarity, there are no studies with solid evidence of superiority of therapeutic strategies. We obtained a good response with rituximab and etoposide, with complete remission of the disease. For this reason, we do not consider the use of the novel monoclonal antibody siltuximab.

\section{Conclusions}

In conclusion, to our knowledge, we report the first case of HHV-8 positive Castleman disease with plasmoblastic microlymphoma occurring in subjects not HIV infected, with a past medical history of KS 
and HBV infection and elevated PCT levels that mimic sepsis during the acute phase of this hematological disease.

\section{References}

1. Fajgenbaum DC, Ruth JR, Kelleher D, et al. The collaborative network approach: a new framework to accelerate Castleman's disease and other rare disease research. Lancet Haematol 2016;3:e150-2.

2. Castleman B, Iverson L, Menendez VP. Localized mediastinal lymph node hyperplasia resembling thymoma. Cancer 1956;9:822-30.

3. Gaba AR, Stein RS, Sweet DL, Varikojis D. Multicentric giant lymph node hyperplasia. Am J Clin Pathol 1978; 69:86-90.

4. Wang HW, Pittaluga S, Jaffe ES. Multicentric Castleman disease: where are we now? Semin Diagn Pathol 2016; 33:294-306.

5. AFIP. Tumors of the lymph nodes and spleen, 4th ed. Washington, DC: AFIP; 2017.

6. Dupin N, Diss TL, Kellam P, et al. HHV-8 is associated with a plasmablastic variant of Castleman disease that is linked to HHV-8-positive plasmablastic lymphoma. Blood 2000;95:1406-12.

7. Diehl S, Rincón M. The two faces of IL-6 on Th1/Th2 differentiation. Mol Immunol 2002;39:531-6.

8. Munshi N, Mehra M, van de Velde H, et al. Use of a claims database to characterize and estimate the incidence rate for Castleman disease. Leuk Lymphoma 2015;56:1252-60.

9. Herrada J, Cabanillas F, Rice L, et al. The clinical behaviour of localized and multicentric Castleman Disease. Ann Intern Med 1998;128:657-62.

10. Nicoli P, Familiari U, Bosa M, et al. HHV8-positive, HIV-negative multicentric Castleman's disease: early and sustained complete remission with rituximab therapy without reactivation of Kaposi sarcoma. Int $\mathbf{J}$ Hematol 2009;90:392-6.
11. van Rhee F, Wong RS, Munshi N, et al. Siltuximab for multicentric Castleman's disease: a randomised,doubleblind, placebo-controlled trial. Lancet Oncol 2014;15: 966-74.

12. Iankova I, Thompson-Leduc P, Kirson NY, et al. Efficacy and Safety of Procalcitonin Guidance in Patients With Suspected or Confirmed Sepsis: A Systematic Review and Meta-Analysis. Crit Care Med 2017 [Epub ahead of print].

13. Nara M, Komatsuda A, Itoh F, et al. Two cases of thrombocytopenia, anasarca, fever, reticulin fibrosis/renal failure, and organomegaly (TAFRO) syndrome with high serum procalcitonin levels, including the first case complicated with adrenal hemorrhaging. Intern Med 2017;56:1247-52.

14. Uldrick TS, Polizzotto MN, Yarchoan R. Recent advances in Kaposi sarcoma herpesvirus-associated multicentric Castleman disease. Curr Opin Oncol 2012;24:495-505.

15. Yuan XG, Chen FF, Zhu YM, et al. High prevalence of hepatitis B virus infection in HIV-negative Castleman's disease. Ann Hematol 2012;91:857-61.

16. Dossier A, Meignin V, Fieschi C, et al. Human herpesvirus 8-related Castleman disease in the absence of HIV infection. Clin Infect Dis 2013;56:833-42.

17. Alkaied H, Harris K, Elsayegh D, et al. Does Kaposi's sarcoma predict multicentric Castleman disease in the presence of generalized lymphadenopathy? Med Oncol 2012;29:1109-13.

18. Koenig G, Stevens TM, Peker D. Plasmablastic microlymphoma arising in human herpesvirus-8associated multicentric Castleman disease in a human immunodeficiency virus-seronegative patient with clinical response to anti-interleukin-6 therapy. Histopathology 2015;67:930-2.

19. Lee YM, Kim JM, Kim SY, et al. Human herpes virus 8/Epstein-barr virus-copositive, plasmablastic microlymphoma arising in multicentric Castleman's disease of an immunocompetent patient. J Pathol Trans Med 2017;51:99-102. 